\title{
Expression of Twist2 is controlled by T-cell receptor signaling and determines the survival and death of thymocytes
}

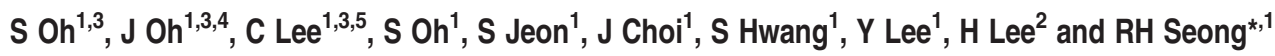

Self-reactive thymocytes are eliminated by negative selection, whereas competent thymocytes survive by positive selection. The strength of the T-cell receptor (TCR) signal determines the fate of thymocytes undergoing either positive or negative selection. The TCR signal strength is relatively higher in negative selection than in positive selection and induces pro-apoptotic molecules such as Nur77 and Nor-1, which are members of the orphan nuclear receptor family, that then cause TCR-mediated apoptosis. However, at the molecular level, it remains unclear how positive or negative selection is distinguished based on the TCR signal. We found that the expression of Twist 2 is differentially regulated in positively and negatively selected thymocytes. In particular, TCR signal strength that elicits positive selection induces Twist2 expression via the $\mathrm{Ca}^{2+}$-Cacineurin-NFATc3 pathway, whereas strength of the TCR signal that results in negative selection abolishes NFATc3-dependent Twist2 induction via specific activation of the JNK pathway. Using Twist2-deficient and Twist2 transgenic mice, we also found that Twist2 determines thymocyte sensitivity to TCR-mediated apoptosis by regulating the expression of Nur77 and Nor-1. Twist2 partially retains histone deacetylase 7 (HDAC7) in the nucleus and recruits it to the Nur77 promoter region to repress Nur77 in positively selected thymocytes. Thus our results suggest a molecular mechanism of how thymocytes interpret the strength of the TCR signal and how TCR sensitivity is controlled during thymic selection.

Cell Death and Differentiation (2016) 23, 1804-1814; doi:10.1038/cdd.2016.68; published online 8 July 2016

T-cells develop from bone marrow-derived progenitors entering the thymus. Initially, immature thymocytes generate and present T-cell receptors (TCRs) on their surface, which recognize the peptide-loaded major histocompatibility complexes (pMHCs) presented by thymic antigen-presenting cells. In order for TCRs to gain infinite reactivity to continually changing pathogens, $\beta$ and $a$ chains of the TCR are sequentially generated through random rearrangement of somatic $\mathrm{V}(\mathrm{D}) \mathrm{J}$ gene segments. Even though this random recombination process confers a great diversity onto the repertoire of TCRs, it also generates unwanted TCRs that are either unreactive or overtly reactive to self-peptide-loaded MHCs (self-pMHCs).

Following the production of surface TCRs, thymic selection eliminates the useless and self-reactive clones while allowing the survival of competent thymocytes. Thymocyte fate is determined by the affinity of their TCRs for self-pMHCs and the resulting strength of delivered signals. Notably, when thymocytes relay TCR signals that are too strong in terms of a high affinity toward self-pMHCs, they induce apoptotic genes, such as Nur77 and Nor-1, and die by 'negative selection'. Only thymocytes possessing TCRs with moderate affinity toward self-pMHCs survive by 'positive selection' and are allowed to differentiate further.

The interaction between a TCR and a self-pMHC in thymocytes activates Ras/mitogen-activated protein kinases, such as ERK and JNK, and the $\mathrm{Ca}^{2+}$-Calcineurin-NFATc3 pathway through a phosphorylation cascade of kinases downstream of TCR. ${ }^{1-5}$ It has been shown that ERK and Calcineurin are activated by the strength of TCR signaling that results in positive selection, whereas JNK activation is triggered only when negative selection strength of TCR signaling is perceived. ${ }^{6}$ Correspondingly, genetic studies in mouse models demonstrate that the ERK and CalcineurinNFATc3 pathways promote positive selection, ${ }^{5,7-10}$ whereas the JNK pathway supports negative selection of thymocytes. ${ }^{11,12}$ However, the downstream events that connect these signaling pathways to their target thymocyte fate are currently not well understood.

During negative selection, Nur77 and Nor-1, which are members of the Nr4a receptor family, are rapidly induced by a

\footnotetext{
${ }^{1}$ School of Biological Sciences and Institute for Molecular Biology and Genetics, Seoul National University, Seoul 151-742, Republic of Korea and ${ }^{2}$ Graduate School of Cancer Science and Policy, National Cancer Center, Gyeonggi 10408, Republic of Korea

${ }^{*}$ Corresponding author: RH Seong, School of Biological Sciences and Institute for Molecular Biology and Genetics, Seoul National University, Seoul 151-742, Republic of Korea. Tel: +82 2 8807567; Fax: +82 2 8879984; E-mail: rhseong@snu.ac.kr

${ }^{3}$ These authors contributed equally to this work.

${ }^{4}$ Current address: Department of Microbiology and Immunology, Sandler Asthma Basic Research Center, University of California San Francisco, San Francisco, CA 94143, USA.

${ }^{5}$ Current address: Hugel, Inc., Chuncheon, Republic of Korea.

Abbreviations: CKO, conditional knockout; CsA, Cyclosporin A; DN, double negative; DP, double positive; HDAC7, histone deacetylase 7; IM, ionomycin; NLC, non-transgenic littermate control; PMA, phorbol-12-myristate-13-acetate; pMHC, peptide-loaded major histocompatibility complex; pre-DP, pre-selected DP; post-DP, post-selected DP; R1, Region 1; R2, Region 2; R3, Region 3; SP, single positive; TCR, T-cell receptor

Received 24.2.16; revised 24.5.16; accepted 08.6.16; Edited by M Piacentini; published online 08.7.2016
} 
strong TCR signal and cause thymocyte apoptosis. ${ }^{13-15}$ Massive cell death of thymocytes is observed in both Nur77 and Nor-1 transgenic mice. ${ }^{13,16,17}$ However, Nur77 null mice do not show any abnormalities in negative selection. ${ }^{18}$ By contrast, negative selection is impaired in mice expressing a dominant-negative form of the Nur77 protein. ${ }^{19}$ It was revealed that these discrepancies are due to the functional redundancy between Nur77 and Nor-1. ${ }^{17,19}$ Therefore, simultaneous control of Nur77 and Nor-1 is indispensable for the regulation of negative selection. The histone deacetylase 7 (HDAC7) has been reported to repress Nur77 expression. ${ }^{20}$ In TCRinexperienced double-positive (DP) thymocytes, nuclear HDAC7 represses Nur77 transcription by interacting with MEF2D, a transcription factor constitutively bound to the MEF2 site in the Nur77 promoter. However, in thymocytes receiving TCR signaling, HDAC7 is released from the Nur77 promoter and exported to the cytoplasm. ${ }^{20-22}$ Subsequently, the empty MEF2 site in the Nur77 promoter is rapidly occupied by $\mathrm{p} 300$ and other transcription co-activators that initiate Nur77 expression. ${ }^{23-25}$ Nevertheless, a recent study showed that HDAC7 is exported to the cytoplasm even during positive selection. ${ }^{26}$ Thus the nuclear/cytoplasmic shuttling of HDAC7 alone is not sufficient to explain the differential expression levels of Nur77 depending on the selected cell fate and suggests involvement of an additional regulator(s).

In this study, we show that Twist2 is induced in thymocytes upon TCR stimulation through the $\mathrm{Ca}^{2+}-$ Calcineurin-NFATc3 pathway. Conversely, specific activation of JNK during negative selection interferes NFATc3-dependent Twist2 induction. TCR-mediated apoptosis of thymocytes is augmented in the absence of Twist2, whereas enforced expression of Twist2 prevents thymocyte apoptosis. Twist2 partially retains HDAC7 in the nucleus of positively selected thymocytes and represses Nur77 expression by facilitating HDAC7 binding to the Nur77 promoter. Thus we demonstrate that Twist2 determines TCR sensitivity in the developing thymocytes.

\section{Results}

Expression of Twist2 is upregulated by TCR signaling in developing thymocytes. Depending on the expression pattern of TCR $\beta$ and CD69, the developmental stages of thymocytes can be divided into three regions: R1 (Region 1), R2 (Region 2), and R3 (Region 3) (Supplementary Figure S1a). These populations are further dissected by the expression pattern of CD4 and CD8: DN (double negative, $\left.\mathrm{CD} 4^{-} \mathrm{CD} 8^{-}\right)$, DP $\left(\mathrm{CD} 4^{+} \mathrm{CD} 8^{+}\right)$, and SP (single positive, $\mathrm{CD} 4^{+} \mathrm{CD}^{-}$or $\mathrm{CD}^{-}{ }^{-} \mathrm{CD} 8^{+}$). In particular, DP cells in the $\mathrm{R} 1$ population are TCR-inexperienced pre-selected DP (pre-DP) cells, whereas DP cells in the R2 population are TCRexperienced post-selected DP (post-DP) cells. Twist2 expression was relatively high at the DN stage and decreased at the pre-DP stage (Figure 1a, Supplementary Figures S1b and $\mathrm{c}$ ). However, it resurged at the post-DP stage and persisted throughout later developmental stages (Figure 1a, Supplementary Figures S1b and c). The induction of Twist2 at the post-DP stage was upregulated at the transcriptional level (Figure 1b).
Expression of Twist2 is induced by the $\mathrm{Ca}^{2+}-$ CalcineurinNFATc3 pathway. To examine whether Twist2 expression is directly regulated by TCR signaling, Twist2 promoter region was cloned and transfected into 16610D9 cells, which are a DP thymoma cell line. ${ }^{27}$ TCR signaling was mimicked by treating with phorbol-12-myristate-13-acetate (PMA) and ionomycin (IM). The promoter activity of Twist2 was efficiently induced by treatment with $0.1 \mathrm{ng} / \mathrm{ml} P M A$ and $200 \mathrm{ng} / \mathrm{ml}$ IM (P0.1/l200) or with $10 \mathrm{ng} / \mathrm{ml} \mathrm{PMA} \mathrm{and} 500 \mathrm{ng} / \mathrm{ml}$ IM (P10/1500) (Figure 1c). Interestingly, the treatment with IM alone (I200 or I500) successfully induced Twist2 promoter activity (Supplementary Figure S2a). IM activates $\mathrm{Ca}^{2+}$ signaling and its downstream components, including Calcineurin and NFAT. Interrupting the $\mathrm{Ca}^{2+}$ signaling by pretreating with Cyclosporin $A(C s A)$, an inhibitor of Calcineurin, completely abolished Twist2 promoter activity after both P0.1/I200 and P10/1500 stimulation (Figure 1c). In addition, the expression of Twist2 was induced in neither thymocytes isolated from the Calcineurin $B 1$ knockout $\left(\mathrm{CnB1}^{-1-}\right)$ nor the NFATC1/NFATC3 double conditional knockout (cKO) (NFATC1, $\mathrm{C3}^{-/}$) mice after stimulation with P0.1/I200 condition (Figure 1d).

The proximal $177 \mathrm{bp}$ of the Twist2 promoter region was sufficient for mediating the PMA/IM response and three putative NFAT-binding sites were detected in this sequence (Supplementary Figures S2b and c). Given that NFATc3 is dominantly expressed among NFAT families at the pre-DP stage, ${ }^{9,28}$ we named these sites c3-1, c3-2, and c3-3, respectively. NFATc3 bound to the c3-1 and c3-3 sites but not to the c3-2 site (Figure 1e). When combinational mutations in the NFAT-binding sites were introduced into the Twist2 promoter, the promoter activity was dependent on the presence of the c3-1 and c3-3 sites but not of the c3-2 site (Figure 1f). When both the c3-1 and c3-3 sites were mutated, NFATc3-dependent augmentation of the Twist2 promoter activity was completely abolished (Figure 1f). In conclusion, TCR signaling induces Twist2 expression via the transcriptional activity of NFATc3.

NFATc3-dependent Twist2 expression is repressed by JNK activity. P0.1/I200 and P10/I500 conditions are experimentally determined to mimic positive and negative selection, respectively. ${ }^{29,30}$ Interestingly, Twist2 promoter activity was lowered in P10/1500 condition (Figure 1c). We thus assumed that Twist2 is differentially regulated during positive and negative selection. To test this possibility, $\mathrm{OVA}_{323-339}$ peptide (OVAp) was injected to the $\mathrm{Rag}^{-/} \times \mathrm{DO} 11.10-\mathrm{TCR}-\mathrm{Tg}$ (hereafter, DO-TCR-Tg) mice to transduce negative selection strength TCR signal. Consistently, Twist2 expression was downregulated by injection with OVAp (Figure 2a). Next we analyzed the temporal kinetics of Twist2 expression depending on the strength of the TCR signal. Unlike the sustained upregulation of Twist2 until $6 \mathrm{~h}$ under the P0.1//200 condition, the Twist2 expression was terminated by $3 \mathrm{~h}$ upon P10/1500 stimulation and remained low thereafter (Figure $2 b$ ). Thus these data suggest that Twist2 expression is repressed by the negative selection strength TCR signal.

NFATc3 localized to the cytoplasm in unstimulated 16610D9 cells (non) and entered the nucleus upon IM stimulation (I200) (Figure 2c). Conversely, the majority of NFATc3 was observed 
a

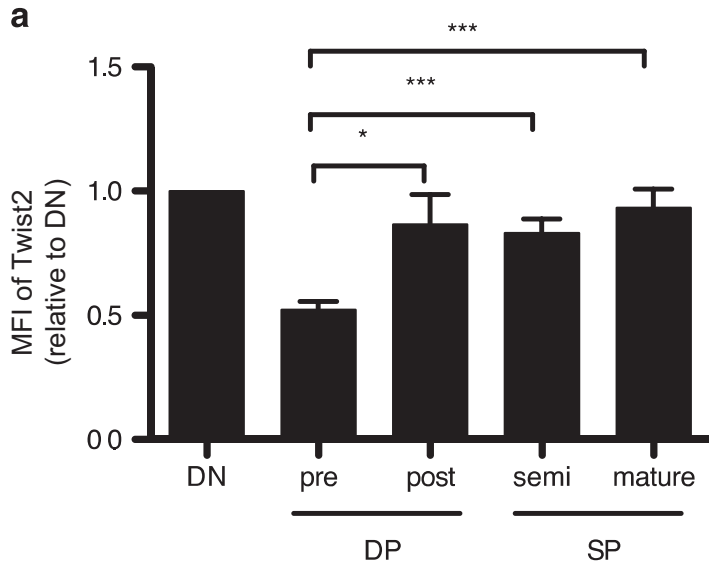

b

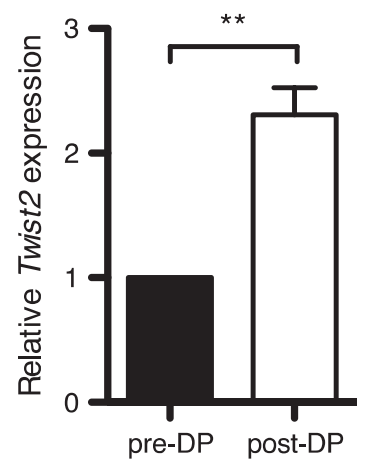

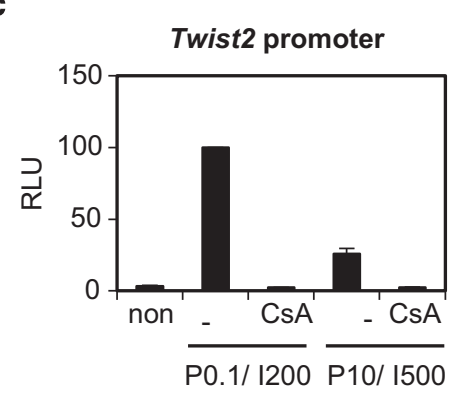

e

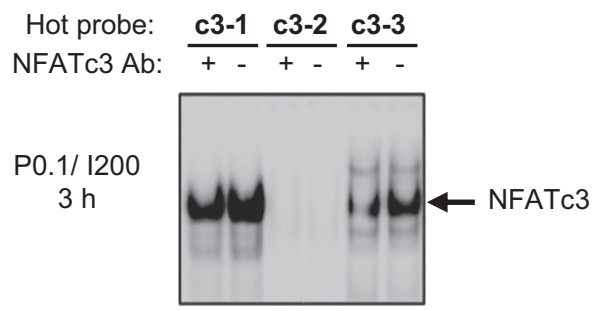

d

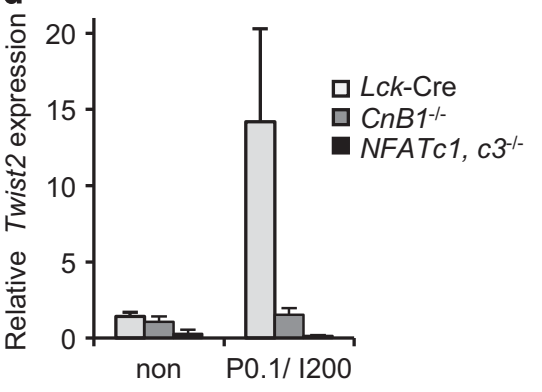

$\mathbf{f}$

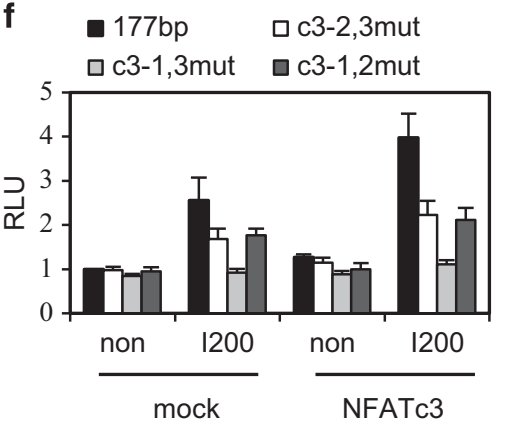

Figure 1 Expression of Twist2 is induced by TCR signal via the $\mathrm{Ca}^{2+}-$ Calcineurin-NFATc3 pathway in developing thymocytes. (a) The expression level of Twist2 was

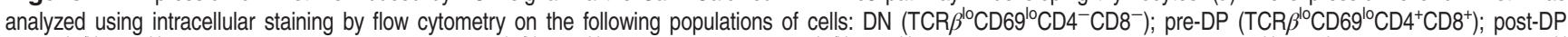
$\left(\mathrm{TCR} \beta^{\text {inthi }} \mathrm{CD} 69^{\text {hi }} \mathrm{CD} 4^{+} \mathrm{CD} 8^{+}\right)$; semimature SP $\left(\mathrm{TCR} \beta^{\text {inthi }} \mathrm{CD} 69^{\text {hi }} \mathrm{CD}^{+} \mathrm{CD} 8^{-}\right.$and $\left.\mathrm{TCR} \beta^{\text {inthi }} \mathrm{CD} 69^{\text {hi }} \mathrm{CD} 4^{-} \mathrm{CD} 8^{+}\right)$; and mature $\mathrm{SP}\left(\mathrm{TCR} \beta^{\text {hi }} \mathrm{CD} 69^{\text {lo }} \mathrm{CD} 4^{+} \mathrm{CD} 8^{-}\right.$and $\mathrm{TCR} \beta^{\text {hi }}$ $\mathrm{CD} 9^{\circ} \mathrm{CD}^{-} \mathrm{CD}^{+}$). Gating strategy is described in Supplementary Figure S1a. Mean fluorescence intensity (MFI) of Twist2 is normalized to lgG, and the results are presented relative to DN stage (error bars \pm S.E.M., $n=9$ ). (b) Twist2 expression in sorted pre-DP and post-DP cells was measured by quantitative PCR (qPCR). The expression of Twist2 is normalized to $\beta$-actin expression, and the results are presented relative to pre-DP stage (error bars, \pm S.E.M., $n=5$ ). (c) $1 \mathrm{~kb}$ Twist2 promoter was transfected to $16610 \mathrm{D} 9$ cells, and the luciferase activities were measured after treatment with combinations with PMA (P0.1, $0.1 \mathrm{ng} / \mathrm{ml}$; and P10, $10 \mathrm{ng} / \mathrm{ml})$ and/or IM (I200, 200 ng/ml; and I500, $500 \mathrm{ng} / \mathrm{ml}$ ). For those indicated below the graph, $5 \mu \mathrm{g} / \mathrm{ml}$ of CsA were treated $15 \mathrm{~min}$ before PMA and IM treatment. The graph presents the relative percentage of luciferase activities to P0.1/l200-treated cells (error bars, \pm S.E.M., $n=3$ ). (d) Twist2 expression in thymocytes from Lck-Cre, CnB1 ${ }^{-1-}$, and NFATc1, $c 3^{-1-}$ mice was measured by qPCR after stimulation with $\mathrm{P} 0.1 / 200$ for $3 \mathrm{~h}$ or not (non). Twist2 expression is normalized to $\beta$-actin expression, and the results are presented relative to not treated (non) thymocytes from Lck-Cre mice (error bars, \pm S.E.M., $n=3$ ). (e) Nuclear extracts from 16610D9 cells were prepared $3 \mathrm{~h}$ after P0.1//200 stimulation. Electrophoretic mobility shift assay was performed on the three putative NFAT-binding sites with the nuclear extracts. Before gel running, anti-NFATc3 antibody was incubated (+) or not (- ). (f) 177 bp or NFAT-binding site mutants of Twist2 promoter constructs were co-transfected with a mock vector (mock) or a NFATc3 expression vector (NFATc3) in 16610D9 cells. After $36 \mathrm{~h}$, cells were further activated with $\mathrm{I} 200$ for $6 \mathrm{~h}$, and the luciferase activities were measured (error bars, \pm S.E.M., $n=3$ )

in the cytoplasm upon IM and PMA co-treatment (P10/I200) (Figure 2c). To verify whether JNK, which is activated by the PMA treatment, exports nuclear NFATc3 to the cytoplasm, cells were pretreated with the JNK inhibitor SP600125 prior to P10/I200 stimulation. When JNK signaling was specifically inhibited, NFATc3 was localized to the nucleus despite
PMA presence (Figure 2c). Consequently, the binding of NFATc3 to the Twist2 promoter was decreased upon P10/1500 stimulation compared with P0.1//200 stimulation (Figure 2d). Thus these results indicate that specific activation of JNK during negative selection interferes NFATc3-dependent Twist2 expression. 
a

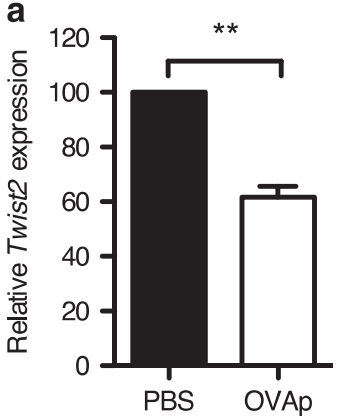

C

Red: NFATc3

Blue: DAPI

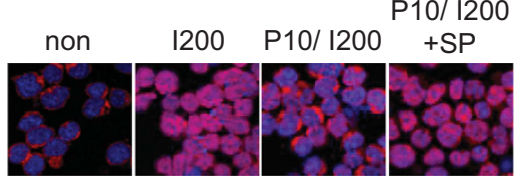

b

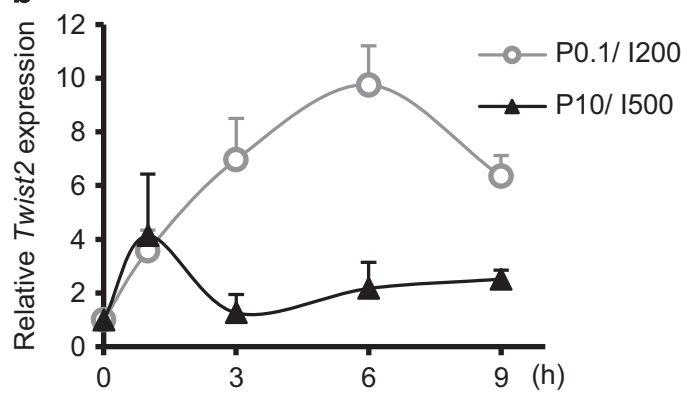

d

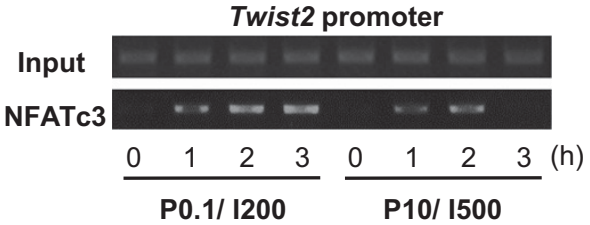

Figure 2 NFATc3-dependent Twist2 expression is repressed by JNK activity. (a) Thymocytes of DO-TCR-Tg mice were isolated at $3 \mathrm{~h}$ after i.p. injection with either PBS or OVAp (100 $\mu$ g). Twist2 expression are presented relative to PBS-injected DO-TCR-Tg mice (error bars, \pm S.E.M., $n=4)$. (b) Thymocytes from Tcr ${ }^{-l-}$ mice were treated with P0.1/1200 or P10/1500 for the indicated time period (0,1,3,6, and $9 \mathrm{~h}$ ). Twist2 expression was measured by qPCR (error bars, \pm S.E.M.). (c) $16610 \mathrm{D} 9$ cells were treated with I0/P0 (non), I200, P10//200, or P10/I200+SP600125 for $3 \mathrm{~h}$. Cells were stained with DAPI (blue) and anti-NFATc3 antibody, which was followed by staining with anti-rabbit-TRITC (red) antibody. (d) ChIP assay was performed by using anti-NFATc3 antibody after stimulation of 16610D9 cells with P0.1//200 and P10/1500 for 0, 1, 2, and $3 \mathrm{~h}$. The eluted supernatants as well as the Input extracts were PCR-amplified with primers for Twist2 promoter in the $-185 \sim+63$ region

TCR-experienced thymocytes are decreased in Twist2deficient mice. To define the role of Twist2 in thymocyte development, Twist $2^{f / f}$ mice were crossed with Cd4-Cre mice. The expression of Twist2 was decreased in thymocytes obtained from Twist $2^{\mathrm{f} / \mathrm{f}} \times \mathrm{Cd}$ 4-Cre $(\mathrm{cKO})$ mice compared with that in Twist2 ${ }^{+/+} \times$Cd4-Cre (control) mice at both the RNA and the protein level (Supplementary Figures S3a-c). We first noticed that the thymic cellularity was slightly reduced in cKO mice compared with control mice (Figure 3a). When we analyzed which developmental stages are reduced in cKO mice, both the percentage and the cell number of the R3 population were decreased in cKO mice compared with control mice (Figures $3 b$ and $c$ ). To validate whether these differences are due to a defect in cell-intrinsic role of Twist2, we conducted mixed bone marrow transfer experiments. Consistently, the percentage of the R3 population was reduced in CD45.2 cKO thymocytes compared with CD45.1 control thymocytes (Supplementary Figures S4a and b).

Interestingly, the percentage of R2 population in CD45.2 cKO thymocytes was significantly reduced compared with CD45.1 control thymocytes (Supplementary Figure S4b). Among the R2 population, the percentage of semimature SP cells, but not post-DP cells, was decreased in both cKO mice and CD45.2 cKO thymocytes (Supplementary Figures S4c and S5a), implying that the significance of Twist2 deletion occurred at the semimature SP stage and enhanced in the competitive environment.

Loss of Twist2 increases the TCR sensitivity to thymocyte apoptosis. Developing thymocytes are more sensitive to TCR stimulation than mature $\mathrm{T}$ cells. ${ }^{31}$ When apoptotic cells were detected in thymocytes, the percentage of Annexin $^{+}$cells was significantly increased in the $\mathrm{R} 3$ population of cKO mice compared with those in control mice (Figure 4a). Intriguingly, the percentage of apoptotic cells in the R2 population was also increased in cKO mice (Figure 4a). However, the significance was comparable in the post-DP population between cKO and control mice (Supplementary Figure S5b), implying that the increased percentage of apoptosis in the $\mathrm{R} 2$ population occurs at the semimature SP stage.

To substantiate whether Twist2 regulates the TCR-mediated apoptosis of thymocytes, thymocytes from control and cKO mice with the DO-TCR-Tg background were co-cultured with MHC-matching $\left(\mathrm{H}-2 \mathrm{~A}^{\mathrm{d}}\right) \mathrm{B}$ cells after pulsing with OVAp. In this system, DO-TCR ${ }^{+}$DP thymocytes undergo apoptosis after recognizing OVAp. ${ }^{32}$ DP cell viability was gradually decreased depending on the dose of OVAp, and it was more significant in cKO mice than that in control mice (Figure 4b). Further, to test directly whether the sensitivity to TCR-induced apoptosis increases in the absence of Twist2, thymocyte apoptosis was measured after treatment with PMA and IM (PMA/IM). TCR-experienced thymocytes were precluded by crossing to the $\mathrm{TCra}^{-1-}$ mice. In cKO thymocytes, PMA/IM-induced apoptosis, but not spontaneous apoptosis, was significantly increased (Figure 4c).

Twist2 inhibits TCR-mediated apoptosis and negative selection of thymocytes. In contrast to the cKO mice, PMA/IM-induced apoptosis of thymocytes was inhibited in Twist2-Tg mice (Figure 5a). We assumed that the discontinued expression of Twist2 is pivotal for the clonal deletion during negative selection. To test this hypothesis, we employed the OT2-TCR-Tg and Rip-mOVA-Tg (hereafter, mOVA-Tg) mouse system and crossed with Twist2-Tg mice. In this system, ovalbumin is expressed in the thymic medulla 
a

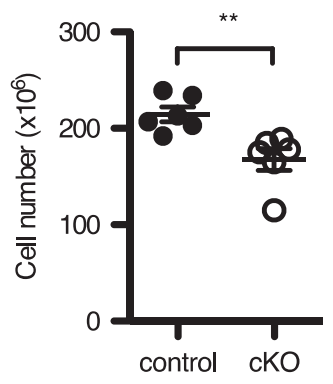

C
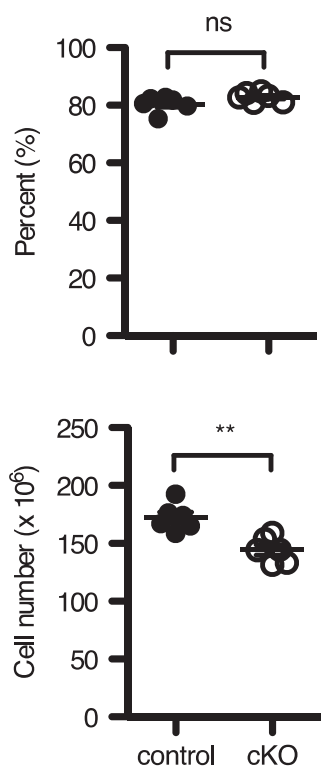

b
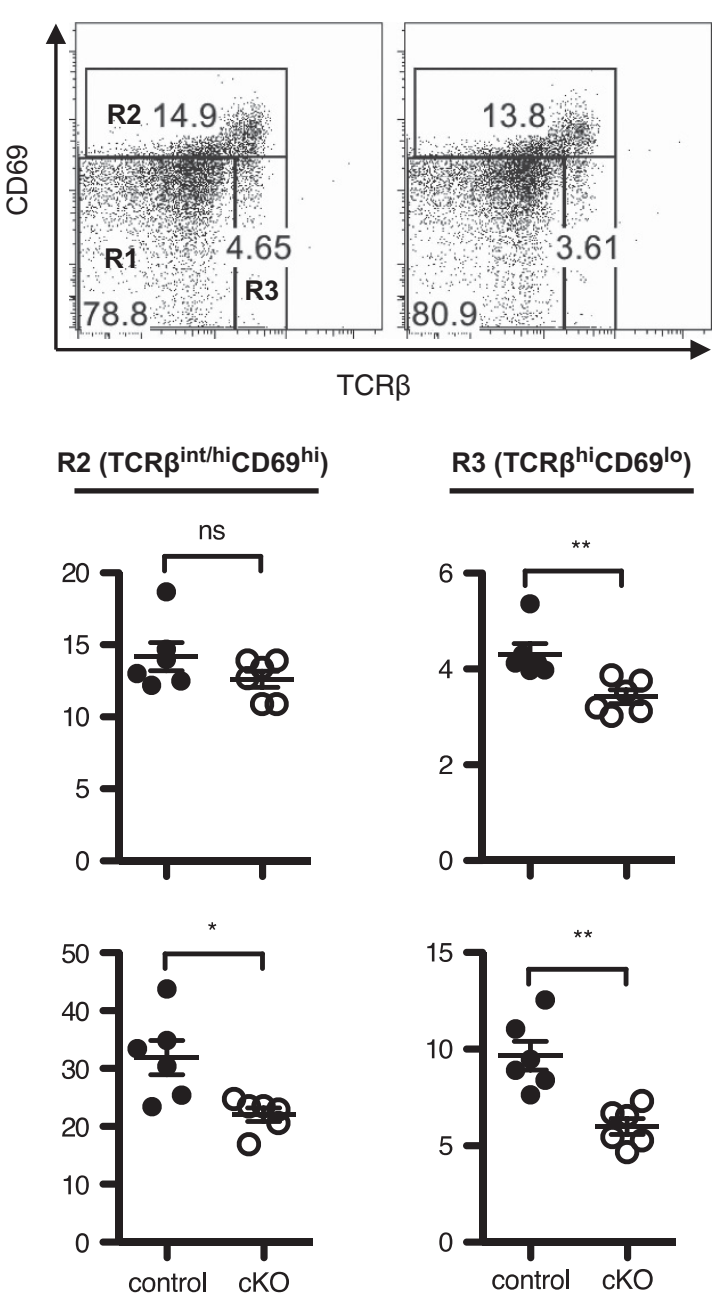

Figure 3 TCR-experienced thymocytes are decreased in Twist2-deficient mice. (a) Thymic cellularity in control and cKO mice is shown (error bars, \pm S.E.M., $n=6$ ). (b) Total thymocytes were stained with anti-CD4, anti-CD8 $\alpha$, anti-TCR $\beta$, and anti-CD69 antibodies. The representative TCR $\beta / C D 69$ plots are shown. (c) The graph presents the

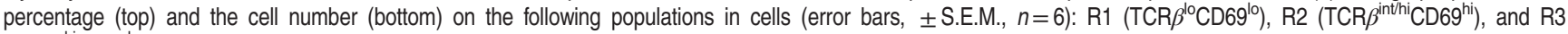
$\left(\mathrm{TCR} \beta^{\mathrm{hi}} \mathrm{CD} 69^{\mathrm{lo}}\right)$ as in panel (b)

and induces negative selection in the $\mathrm{CD} 24^{\mathrm{lo}} \mathrm{V} a 2^{\text {hi }}$ population (Figures $5 b-d) .{ }^{33}$ Unlike the dramatic reduction of both total thymic cellularity and the percentage of the $\mathrm{CD} 24^{\mathrm{lo}} \mathrm{Va} 2^{\mathrm{hi}}$ population in non-transgenic littermate control (NLC) mice, negative selection was impaired in Twist2-Tg mice (Figures 5b-d).

Notably, thymic cellularity in OT2-TCR $\times$ Twist2-Tg mice was decreased compared with OT2-TCR-Tg mice (Figure 5c). The reduced thymic cellularity in Twist2-Tg mice might be influenced by a yet unexplored role of Twist2 at the DN stage as inferred from the increased DN population in OT2-TCR $\times$ Twist2-Tg mice (Figure 5b). As only the CD4 SP cells in the $\mathrm{CD} 24^{\mathrm{lo}} \mathrm{Va} 2^{\text {hi }}$ population are eliminated by negative selection in OT2-TCR $\times$ mOVA-Tg mouse system, we calculated the fold reduction of CD4 SP cells to measure the efficiency of negative selection specifically. As expected, the efficiency of negative selection in CD4 SP cells was lower in Twist2-Tg mice than that in NLC mice (NLC: $73.88 \pm 5.218 \%$ versus Twist2-Tg: 49.78 $\pm 4.681 \%$ ) (Supplementary Figure S6).
Twist2 regulates the expression of Nur77 and Nor-1. Nur77 is required for the negative selection in OT2-TCR $\times$ mOVA-Tg mice system. ${ }^{33,34}$ To determine whether Twist2 regulates Nur77 induction in this system, we measured Nur77 levels in the $\mathrm{CD} 24^{\mathrm{lo}} \mathrm{Va} 2^{\text {hi }} \mathrm{CD} 4^{+}$population (Figure $6 \mathrm{a}$ ). In OT2-TCR $\times$ mOVA-Tg mice, both the percentage of Nur $77^{\text {hi }}$ cells and the expression of Nur77 were significantly increased compared with OT2-TCR-Tg mice (Figures 6a-c). However, Nur77 induction was attenuated in the presence of Twist2-Tg strain (Figures 6a-c). To assess whether Twist2 regulates Nur77 expression at the transcriptional level, the expression of Nur77 and its related gene, Nor-1, was measured in thymocytes obtained from NLC and Twist2-Tg mice after PMA/IM treatment. Consistently, the induction of Nur77 and Nor-1 was repressed in Twist2-Tg mice (Figure 6d). Conversely, the expression of Nur77 and Nor-1 was significantly increased in cKO mice compared with control mice (Figure 6e). 
a
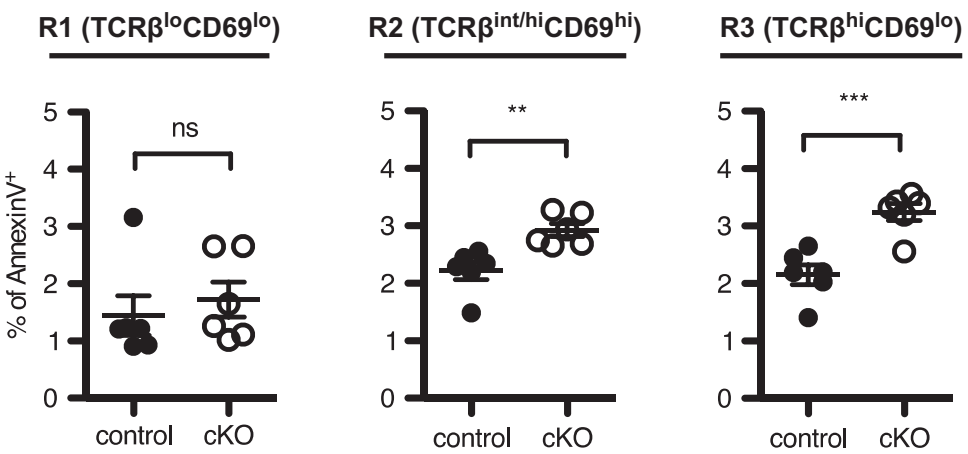

b

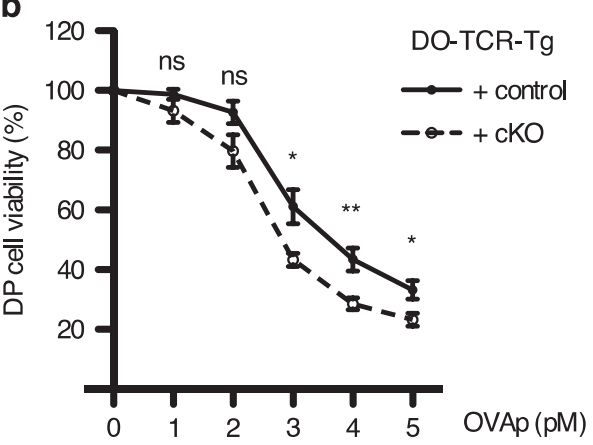

C
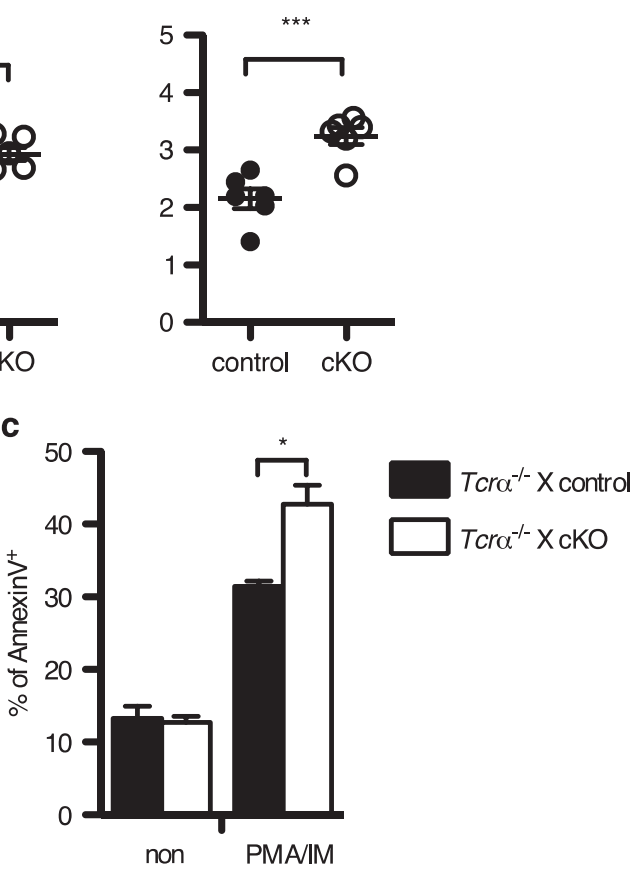

Figure 4 Loss of Twist2 increases the sensitivity of thymocytes to TCR-mediated apoptosis. (a) The percentage of AnnexinV ${ }^{+}$cells in TCR $/$/CD69 subpopulations as in Figure $3 \mathrm{c}$ is summarized (error bars, \pm S.E.M., $n=6$ ). (b) The percentage of DP cells in thymocytes obtained from DO-TCR-Tg mice was detected at $24 \mathrm{~h}$ after co-culture with OVAp (OVA ${ }_{323-339}$ peptide) pre-loaded B cells using FACS Cantoll. The graph presents the relative percentage of DP cells to non-stimulated cells (error bars, \pm S.E.M., $n=6$ ). (c) Indicated mice were crossed with $\mathrm{Tcr}^{-1-}$ mice to preclude TCR-experienced thymocytes. Apoptotic cells were detected after PMA and IM stimulation using AnnexinV staining kit. The graph presents the AnnexinV $V^{+}$percentage in control and cKO mice (error bars, \pm S.E.M., $n=3$ )

To examine whether Twist2 directly regulates the expression of Nur77 and Nor-1, the promoter region of Nur77 and Nor-1 was cloned and the promoter activities were detected in 16610D9 cells after PMA/IM stimulation (Figure 6f). Twist2 repressed the promoter activities of Nur77 and Nor-1 (Figures $6 \mathrm{~g}$ and $\mathrm{h}$ ). The MEF2D-binding site (MEF2 site) and the HDAC7-binding site (HDAC7 site) have been reported in each promoter region. ${ }^{15,23,35}$ Twist2 bound to the MEF2 site in the Nur77 promoter and the HDAC7 site in the Nor-1 promoter (Figure 6i). To validate whether Twist2 regulates the activities of Nur77 and Nor-1 promoters at these sites, we prepared mutant promoter constructs (Nur77-mutMEF2 and Nor-1-mutHDAC7). Unlike intact Nur77 and Nor-1 constructs, Twist2 failed to repress the mutant promoters (Figures $6 \mathrm{~g}$ and $\mathrm{h}$ ).

Twist2 interacts with MEF2D and HDAC7 to repress the expression of Nur77 and Nor-1. Next we tested whether Twist2 physically associates with MEF2D and HDAC7. To detect protein-protein interaction, we conducted a bimolecular fluorescence complementation (BiFC) analysis. ${ }^{36}$ In this system, if two proteins interact, the combined VN/VC (VENUS) emits green fluorescence. It was previously reported that $\mathrm{MEF} 2 \mathrm{C}$, which shares a highly conserved sequence with MEF2D, interacts with full-length Twist2, but not with a C-terminal-deleted form of Twist2 (Twist2C $\Delta){ }^{37}$ Consistently, full-length Twist2, but not Twist2C $\Delta$, interacted with MEF2D (Figure 7a). HDAC7 was also associated with full-length Twist2, but not with Twist2C $\Delta$, in both BiFC and co-immunoprecipitation analysis (Figure $7 \mathrm{a}$ and Supplementary Figure S7).

As Twist2 interacted with MEF2D and HDAC7, we deduced that Twist2 enhances the binding of HDAC7 to the Nur77 promoter. Twist2 recruited HDAC7 to the Nur77 promoter regardless of $\mathrm{PMA} / \mathrm{IM}$ stimulation, whereas it prevented the binding of p300 (Figures $7 \mathrm{~b}$ and $\mathrm{c}$ and Supplementary Figure S8a). Although HDAC7 is mainly detected in the cytoplasm of positively selected thymocytes, ${ }^{26}$ nuclear HDAC7 in DP thymocytes was higher in OT2-TCR $\times$ Twist2-Tg mice than that in OT2-TCR-Tg mice (Figure 7d). In addition, Twist2 inhibited the nuclear export of HDAC7 in 16610D9 cells upon TCR stimulation (Supplementary Figure S8b). To delineate whether Twist2 represses the Nur77 expression through the physical interactions with MEF2D and HDAC7, the promoter activity of Nur77 was measured in the presence of Twist $2 \mathrm{C} \Delta$. In contrast to full-length Twist2, Twist2C $\Delta$ was neither able to repress the Nur77 promoter activity nor induce apoptosis in 16610D9 cells upon PMA/IM stimulation (Figures $7 e$ and f).

\section{Discussion}

In this study, we show that Twist2 is differentially regulated depending on the strength of TCR signaling and determines TCR sensitivity of developing thymocytes. At the downstream of proximal TCR signaling, Twist2 expression is upregulated by the $\mathrm{Ca}^{2+}$-Calcineurin-NFATc3 pathway in positively selected thymocytes, whereas it is repressed by the JNK pathway, which is 

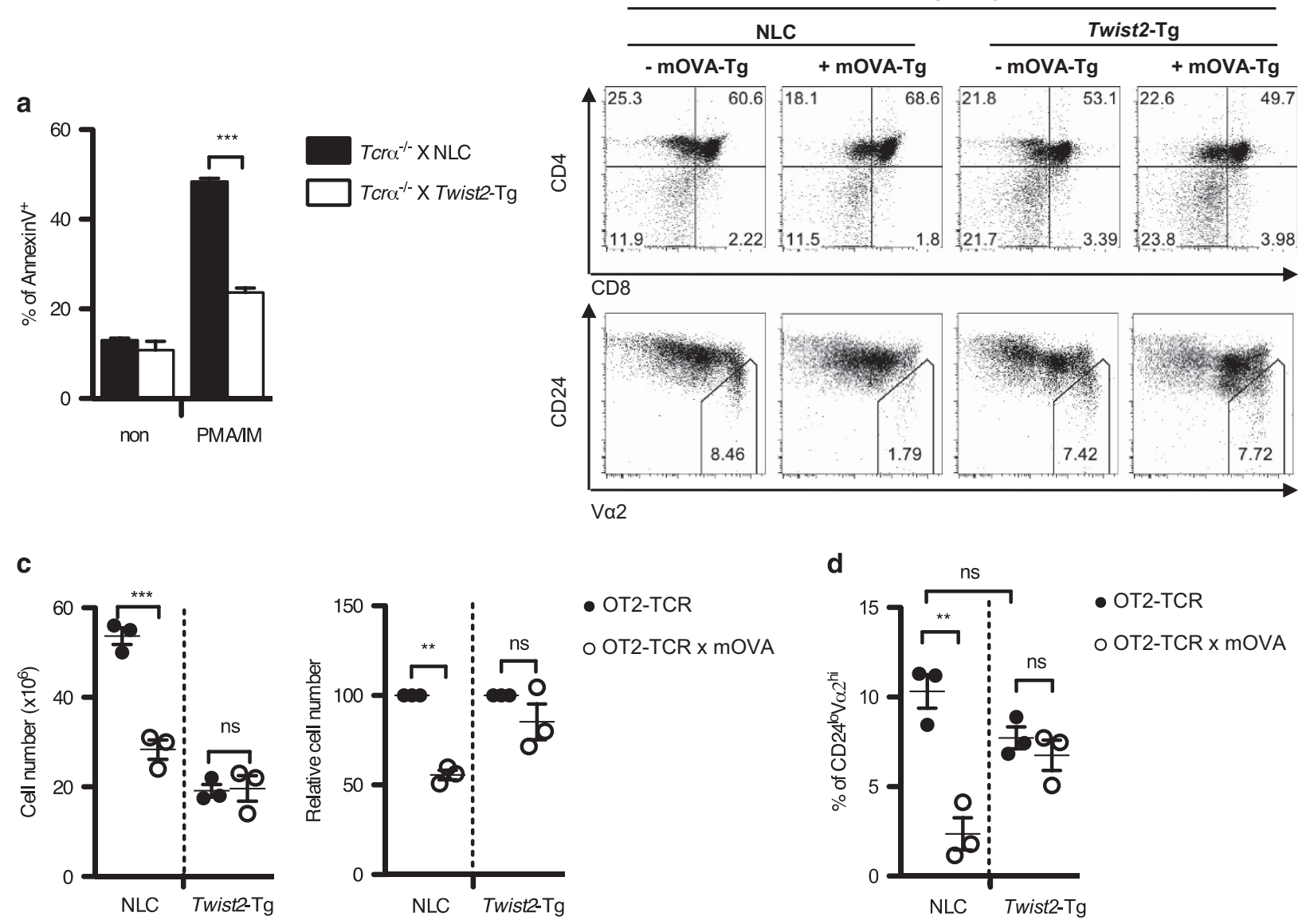

Figure 5 Twist2 inhibits TCR-mediated apoptosis and negative selection of thymocytes. (a) Indicated mice were crossed with $\mathrm{Tcr}^{-1-}$ mice. Apoptotic cells were detected after PMA and IM stimulation using AnnexinV staining kit. The graph presents the AnnexinV ${ }^{+}$percentage in NLC and Twist2-Tg mice (error bars, \pm S.E.M., $n=3$ ). (b) Representative CD4/CD8 and CD24/N $\alpha 2$ FACS plots of the indicated mice. (c) The graph presents the total thymic cellularity in the indicated mice (left). Relative percentage of thymic cellularity in OT2-TCR $\times$ mOVA-Tg mice and OT2-TCR-Tg mice with either NLC or Twist2-Tg mice is shown (right) (error bars, \pm S.E.M., $n=3$ ). (d) The percentage of the $\mathrm{CD} 24^{10} \mathrm{~V} \alpha 2^{\text {hi }}$ population in total thymocytes from the indicated mice is summarized (error bars, \pm S.E.M., $n=3$ )

specifically activated in negatively selected thymocytes. In addition, TCR sensitivity of thymocytes is determined depending on the expression level of Twist2. Hence, it seems that Twist2 determines the fate of thymocyte, either positive or negative, selection at the downstream of TCR signaling.

It is known that the $\mathrm{Ca}^{2+}$-Calcineurin signal is preferentially required for positive selection and that the activated Calcineurin dephosphorylates NFATc3 to allow its translocation into the nucleus. ${ }^{5}$ On the contrary, it has also been reported that activation of the JNK pathway inhibits transcriptional activity of NFATc3 by exporting NFATc3 to the cytoplasm. ${ }^{38}$ However, the physiological significance of JNK-mediated export of NFATc3 has not been elucidated. We found that Twist2 expression was reciprocally regulated by NFATc3 and JNK. Under the strength of TCR stimulation that induces positive selection, NFATc3 bound to the Twist2 promoter and activated the promoter activity. By contrast, activated JNK pathway upon negative selection strength of TCR stimulation interfered with the NFATc3-mediated Twist2 expression. Thus here we suggest a model whereby thymocytes translate the strength of TCR signaling into Twist2 expression.
Based on the low expression of Nur77 in positively selected thymocytes, it is expected that localization of HDAC7 would be nuclear in these cells. However, HDAC7 in mainly detected in the cytoplasm of positively selected thymocytes. ${ }^{26}$ Interestingly, however, Twist2 partially sequestered HDAC7 in the nucleus of activated 16610D9 cells and positively selected DP thymocytes. Moreover, Twist2 could recruit HDAC7 to the Nur77 promoter. The remaining question is how Twist2 recruits HDAC7 to the Nur77 promoter. Our current work suggests that Twist2 can physically interact with both MEF2D and HDAC7. Subsequently, Twist2 recruits HDAC7 on the Nur77 promoter while preventing the binding of $\mathrm{p} 300$ to the same site. Thus it seems that Twist2 modulates the binding abilities of transcriptional activators and repressors on the Nur77 promoter depending on the strength of the TCR signal.

Another interesting finding is that Twist2 can simultaneously regulate the expression of Nur77 and Nor-1. It has been reported that Nor-1 has a functional redundancy with Nur77 during negative selection of thymocytes. ${ }^{17-19} \mathrm{HDAC} 7$ mutant mice (HDAC7 $\triangle \mathrm{P}-\mathrm{Tg}$ ), where HDAC7 lost its nuclear export property, show decreased expression of Nur77 and Nor-1. ${ }^{34}$ These results imply a possible role for HDAC7 in the 
a

目 OT2-TCR x mOVA $\square$ ㅁ OT2-TCR

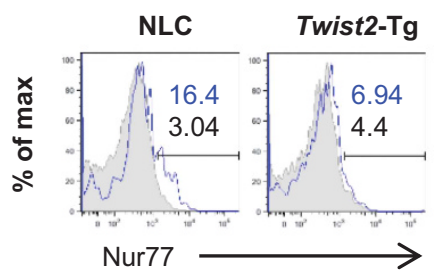

b

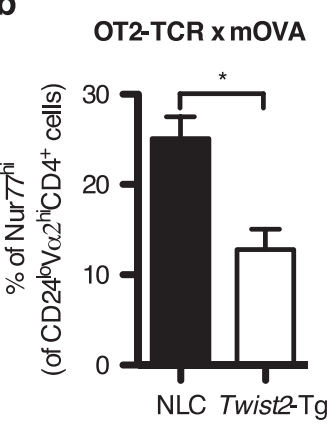

C

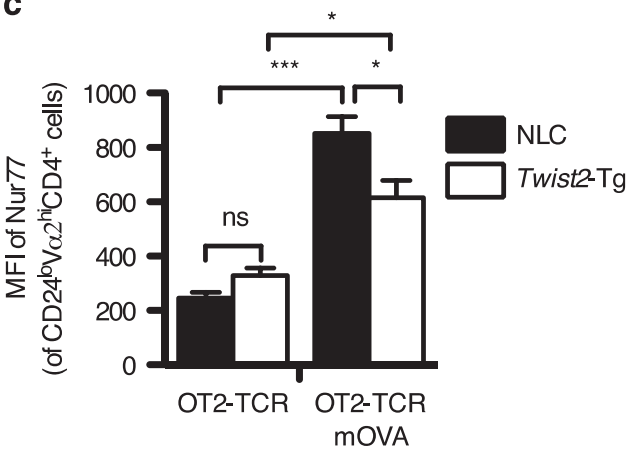

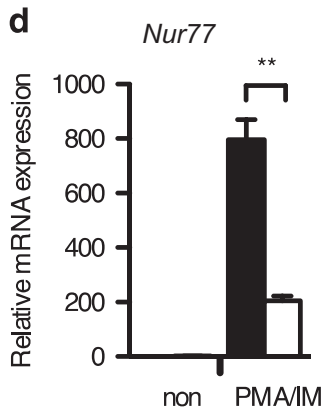

non PMAIM
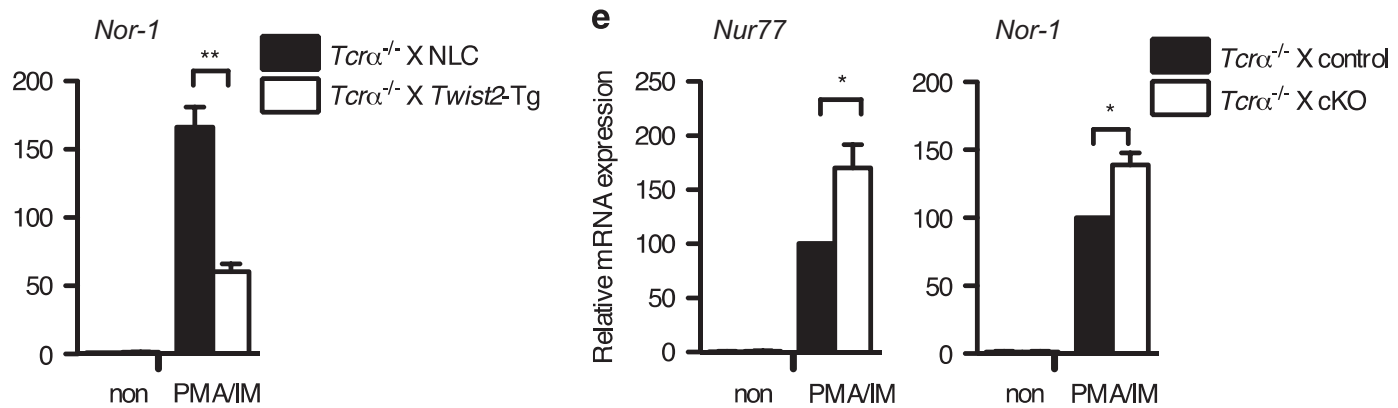

$\mathbf{f}$

pGL3-Nur77

pGL3-Nur77-mutMEF2

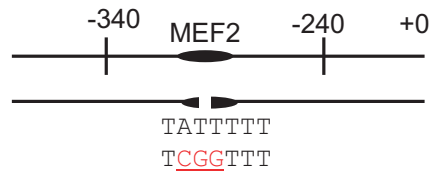

pGL3-Nor-1

pGL3-Nor-1-mutHDAC7

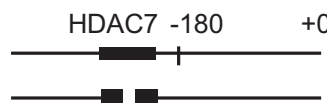

CGGAGTTTCC

CAAAGTTTCC $+0$

h

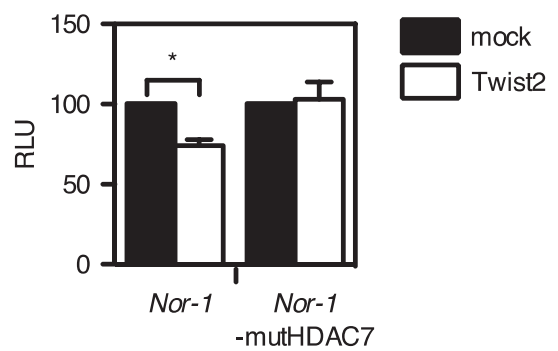

g

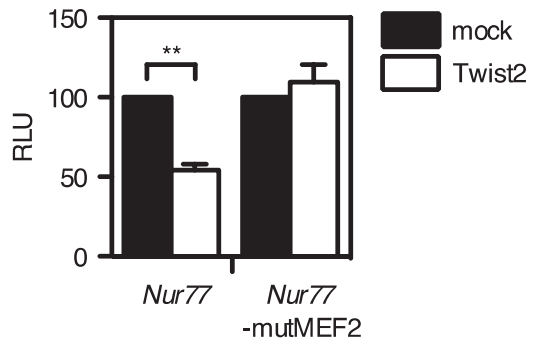

i

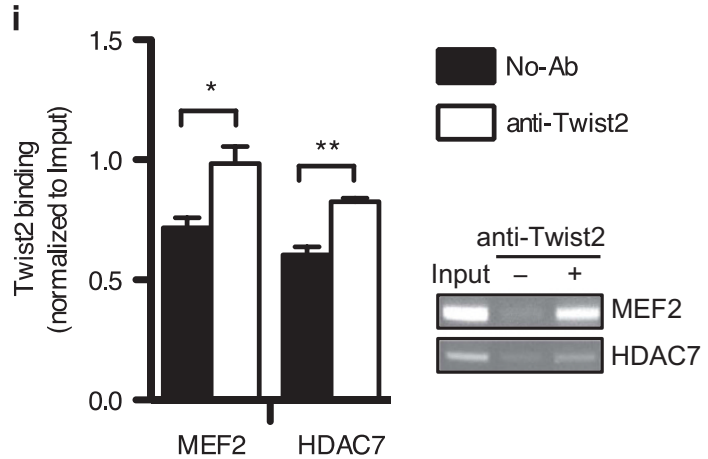

Figure 6 Twist2 regulates the expression of Nur77 and Nor-1. (a-c) The expression level of Nur77 in the CD2 $4^{\text {lo }} \mathrm{V} \alpha 2^{\text {hi }} \mathrm{CD} 4^{+}$population was analyzed by flow cytometry using intracellular staining. Numbers indicate the percentage of Nur $77^{\text {hi }}$ cells in each population (blue solid line: OT2-TCR $\times$ mOVA-Tg, gray tinted line: OT2-TCR-Tg) (a). The percentage of Nur77 $7^{\text {hi }}$ cells (b) and the MFI of Nur77 (c) in the indicated mice are summarized (error bars, \pm S.E.M., $n=3$ ). (d and e) The expression of Nur77 and Nor-1 was detected using GPCR in thymocytes from NLC and Twist2-Tg mice (d) or control and cKO mice (e) after stimulation with PMA and IM (PMA/IM). All mice were crossed with $\mathrm{TCr}^{-1-}$ mice to preclude TCR-experienced thymocytes. The expression of Nur77 and Nor-1 expression is normalized to $\beta$-actin expression, and the results are presented relative to non-stimulated respective control thymocytes (error bars, \pm S.E.M.). (f) Schematic diagrams of the Nur77 and the Nor-1 promoter constructs used in luciferase assay. Mutated sequences in each promoter are indicated in red color. ( $\mathbf{g}$ and $\mathbf{h}$ ) Twist2 expression vector was co-transfected into $16610 \mathrm{D} 9$ cells with Nur77 and Nur77-mutMEF2 promoter constructs (g) or Nor-1 and Nor-1-mutHDAC7 promoter constructs (h). Luciferase activities were detected after the PMA/IM treatment. The results are presented relative to the promoter activity in mock-transfected cells (error bars, \pm S.E.M.). (i) ChIP assay was performed in thymocytes from DO-TCR $\times$ Twist2-Tg mice. The binding of Twist2 to the promoters of Nur77 and Nor-1 was detected by semi-qPCR. The band intensity was quantified using the ImageJ software (National Institutes of Health, Bethesda, $\mathrm{MD}, \mathrm{USA}$ ) and normalized to Input (error bars, \pm S.E.M.) 
a

MEF2D-VN MEF2D-VN HDAC7-VN HDAC7-VN Twist2-VC Twist2C $\Delta$-VC Twist2-VC Twist2C $\Delta$-VC
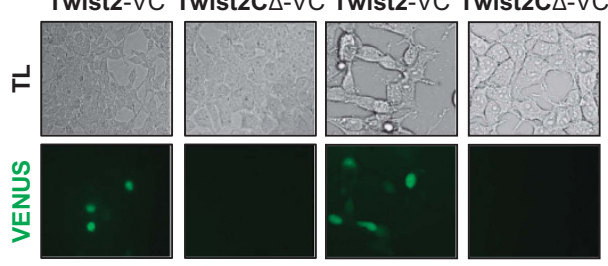
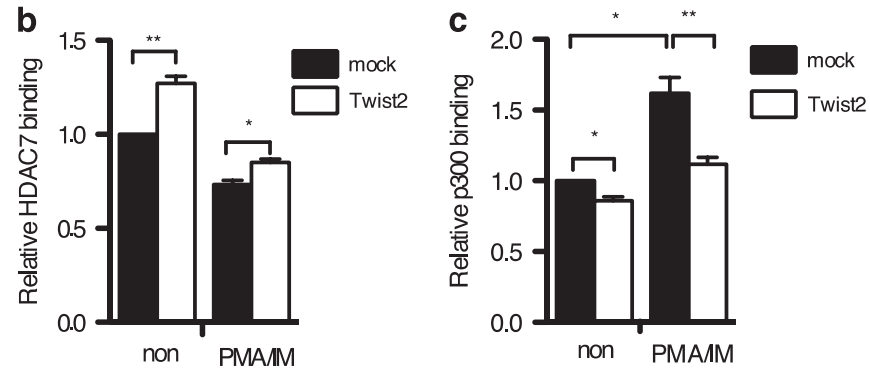

d

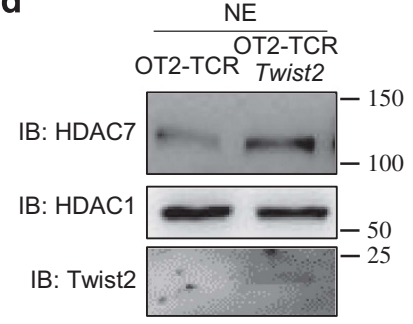

e

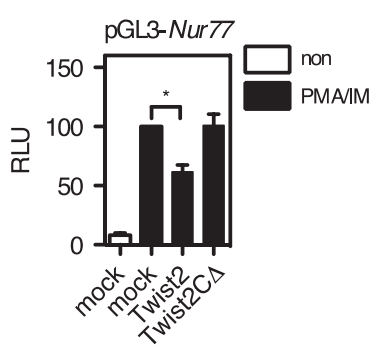

f

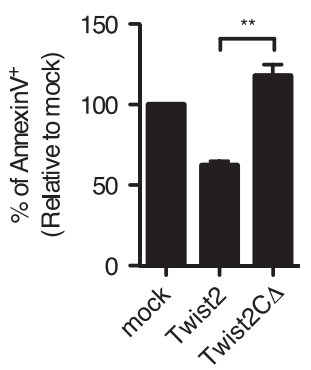

Figure 7 Twist2 interacts with MEF2D and HDAC7 to repress the expression of Nur77 and Nor-1. (a) Twist2, Twist2C $\Delta$, MEF2D, and HDAC7 were cloned into either VN-173 or VC-155 vector. Indicated VN/VC vector combinations were transfected into 293T cells, and fluorescence was detected after $48 \mathrm{~h}$ using fluorescence microscope (LSM 710 ). (b and c) 16610D9 cells were transfected with either a mock or a Twist2 expression vector. The binding of HDAC7 (b) or p300 (c) was detected by ChIP assay after PMA and IM stimulation. The binding of HDAC7 and p300 was quantified using the ImageJ software and normalized to Input. The graphs present the relative bindings to non-stimulated mock (error bars, \pm S.E.M.). (d) HDAC7 was detected in the nuclear extract (NE) in sorted OT2-TCR ${ }^{+}$DP thymocytes obtained from OT2-TCR-Tg mice and OT2-TCR $\times$Twist2-Tg mice by western blotting. (e) The Nur77 promoter construct was co-transfected with either a Twist2 or a Twist2C $\Delta$ vector in $16610 \mathrm{D} 9$ cells. Transfected cells were activated $1 \mathrm{~h}$ with PMA/IM or not treated (non). Relative luciferase activities to PMA/IM-treated mock are shown (error bars, \pm S.E.M.). (f) $16610 \mathrm{D} 9$ cells were transfected with either a Twist2 or a Twist2 $\mathrm{C} \Delta$ vector and apoptotic cells were detected using AnnexinV staining after PMA and IM stimulation for $6 \mathrm{~h}$. The graph presents the relative percentage of AnnexinV ${ }^{+}$cells to mock-transfected cells (error bars, \pm S.E.M.)

expression of Nor-1 as well as Nur77. Unlike the Nur77 promoter where MEF2D has a role as an anchor protein for HDAC7, no MEF2D-binding elements have been reported in the Nor-1 promoter region. It is yet unclear whether Twist2 represses Nor-1 expression in a MEF2D-dependent manner. Nevertheless, we found that Twist2 bound to the same region of Nor-1 promoter as HDAC7 and that Twist2 promoted the HDAC7 binding to the Nor-1 promoter. Therefore, a Twist2/ HDAC7 complex is crucial for the simultaneous regulation of Nur77 and Nor-1 expression.

It was previously reported that the secretion of TNFa is increased in Twist2-null mice and Twist ${ }^{-1-}$ cells are sensitive to TNFa-induced apoptosis. ${ }^{39}$ As TNFa is constitutively expressed in the thymus, ${ }^{40}$ it is possible that the elevated apoptosis of thymocytes in the cKO mice is also due to their increased sensitivity to TNFa-induced apoptosis. However, TNFa-induced apoptosis is mostly restricted to the DN stage cells and negative selection does not appear to be affected by TNFa. ${ }^{41,42}$ In addition, Twist2 was deleted in DP cells after the DN stage in the cKO mice. Twist2 may also have a role in thymocyte development together with E-proteins, as it forms a heterodimer with E-proteins via the bHLH domain. ${ }^{37,43}$ Downregulation of E-protein activity during DP-to-SP transition is crucial for thymic selection because mice with mutated Id3, another E-protein-binding partner that lacks a DNAbinding domain, displayed abnormalities in positive and negative selection. ${ }^{44}$ Thus it is conceivable that abnormal E-protein activity in cKO mice may increase thymocyte apoptosis even in the presence of functional Id3. However, Twist2C $\Delta$ protein, which still has the ability to form a heterodimer with E-proteins, failed to repress TCR-mediated apoptosis. Of note, it has also been reported that Twist2 functions as a repressor by interacting with transcription factors other than E-proteins. ${ }^{39,45}$ Therefore, even though there is a possibility of a partial contribution of Twist2/E-protein heterodimers to thymic selection, it is more likely that the increased apoptosis during thymic selection in cKO mice is due to enhanced TCR sensitivity caused by the disruption of the Twist2/HDAC7 complex on the promoters of Nur77 and Nor-1, which is independent from E-protein activity.

Nur77 was reported to be expressed in positively selected thymocytes, although its expression level is one-fifth in induction-fold compared with that of negatively selected cells. ${ }^{46,47}$ However, it was also reported that positive selection was normal in the Nur77-deficient mice. ${ }^{18,48}$ Recently, enhanced positive selection was reported in Nur77 and Bim double knockout mice. ${ }^{48}$ It seems that the veiled function of Nur77 in positive selection was revealed only when Nur77 was disrupted simultaneously with Bim. We have shown that Twist2 represses the expression of Nur77. However, Twist2 does not appear to regulate the Bim expression (data not shown). Therefore, our results that no significant change in positive selection occurred in Twist2-Tg mice seem to be consistent with the previous results that Nur77 deficiency alone has a minimal effect in positive selection. ${ }^{48}$ 
Nur77 and Nor-1 have a crucial role in thymocyte apoptosis upon TCR signaling. Consequently, the abnormal regulation of Nur77 and Nor-1 expression leads to impaired thymocyte development, which ultimately results in an unhealthy immune system. Despite the importance of Nur77 and Nor-1, the transcription factor(s) that is differentially regulated by TCR signaling and regulates Nur77 and Nor-1 expression has not been identified yet. Our study provides evidences that Twist2 is discriminatively regulated depending on the intensity of the TCR signal and determines the expression level of Nur77 and Nor-1 in cooperation with HDAC7. Thus Twist2 appears to be a pivotal regulator of survival and death in developing thymocytes.

\section{Materials and Methods}

Mice. A cDNA encoding mouse Twist2 was obtained by reverse transcription-PCR (RT-PCR) from thymocyte RNA preparations and was cloned into the BamHI site of the p1017 vector under the control of a mouse Lck proximal promoter by using conventional cloning procedures. ${ }^{49}$ Twist2 transgenic mice were obtained essentially as described previously..$^{50}$ To specifically delete Twist2 in thymocytes, Twist $2^{\text {t/ }}$ mice were crossed with Cd4-Cre-Tg mice. $\mathrm{Tcr}^{-1-}$, OT2-TCR-Tg, and Rip-mOVA-Tg mice were purchased from Jackson Laboratory (Bar Harbor, ME, USA). $\mathrm{Rag}^{-1-} \times \mathrm{DO} 11.10 \mathrm{TCR}-\mathrm{Tg}$ mice were purchased from Taconic Farms (Germantown, NY, USA). The mice were bred and maintained under specific pathogen-free condition, and experiments were performed in accordance with institutional and national guidelines.

Flow cytometric analysis and cell sorting. Thymocytes were harvested, stained, and analyzed on a FACSCanto II (BD Biosciences, San Jose, CA, USA). Antibodies with the following specificities were used for staining: anti-CD69-PerCP, anti-V $\alpha 2$-biotin, anti-TCR $\beta$-APC, anti-CD4-PE, anti-CD4-PEcy7, anti-CD8 $\alpha$-APCcy7, anti-CD24-PE-cy7, anti-CD24-FITC, anti-D011.10-TCR-PerCP, and anti-StreptavidinAPC (all from BD Biosciences or eBioscience (San Diego, CA, USA)). Where indicated, thymocytes were sorted into $\mathrm{TCR} /{ }^{\text {lo }} \mathrm{CD} 69^{\mathrm{lo}} \mathrm{CD} 4^{+} \mathrm{CD} 8^{+}$(pre-DP) and $\mathrm{TCR} \beta^{\text {inth }} \mathrm{CD} 69^{\text {hi }} \mathrm{CD} 4^{+} \mathrm{CD} 8^{+}$(post-DP) subpopulations on a FACSAria \|| (BD Biosciences). The purity of all sorted population was $>95 \%$.

DP cell viability assay, AnnexinV staining, and intracellular staining. Untouched B cells were sorted using Dynabeads (Invitrogen, Carlsbad, CA, USA) following the manufacturer's instruction. Briefly, splenocytes of BALB/C mice were mixed with Dynabeads and biotin-conjugated anti-CD4, anti-CD8, antiTer119, anti-CD3e, anti-CD11b, and anti-GR1 antibodies for $20 \mathrm{~min}$ at RT. Supernatants containing bead-free untouched $B$ cells were separated using magnet and collected into a new tube. DP cell viability was detected by gating D011.10 $\mathrm{TCR}^{+} \mathrm{CD} 4^{+} \mathrm{CD}^{+}$population after incubation for $24 \mathrm{~h}$ with untouched $\mathrm{B}$ cells. OVA $_{323-339}$ (ISQAVHAAHAEINEAGR) peptide (GL Biochem, Shanghai, China) was preloaded before $30 \mathrm{~min}$ to untouched $B$ cells prior to the incubation with thymocytes. For AnnexinV staining, thymocytes and 16610D9 cells were activated with PMA (LC laboratories, Woburn, MA, USA) and IM (LC Laboratories), and apoptosis was detected using the AnnexinV-FITC Apoptosis Kit (BD Biosciences) according to the manufacturer's protocol. For intracellular staining, fleshly isolated thymocytes were stained with anti-CD4-PEcy7, anti-CD8-APCcy7, anti-TCR $\beta$-APC, anti-CD69-PerCP, anti-CD24-FITC, anti-V $\alpha 2-$ Biotin, and anti-Streptavidin-APC antibodies. Stained thymocytes were fixed and further stained with anti-lgG, antiTwist2, or anti-Nur77-PE antibodies using the Foxp3 Fix/Perm Kit (eBioscience) following the manufacturer's instruction. To detect intracellular Twist2, anti-lgG-PE antibody was further added.

Cell culture and luciferase assay. Primary thymocytes were cultured on RPMI medium with 10\% FBS and 2-mercaptoethanol. 16610D9 cells were maintained with Opti-MEM (Invitrogen, Carlsbad, CA, USA) supplemented with $10 \%$ FBS and 2-mercaptoethanol. For luciferase assay, the promoters of Nur77 and Nor-1 and the Twist2 promoter were obtained from genomic DNA of Jurkat T cell line and c57BL/6 mouse, respectively, and cloned into pGL3-basic vector. Point mutations in each promoter constructs were generated by site-directed mutagenesis as instructed (Stratagene, La Jolla, CA, USA). ${ }^{51}$ Designated vectors in each experiment were transfected in 16610D9 cells. Twenty four-hours later, cells were treated with PMA/IM and luciferase activities were detected using the Luciferase Assay Kit (Promega, Madison, WI, USA) as instructed in manual. CsA (Sigma, St. Louis, MO, USA) was treated 15 min before the treatment of PMA and IM.

Semiquantitative and quantitative PCR. RNA was extracted from the sorted populations or cells by Trizol reagent (Invitrogen) and then used for reverse transcription. Quantitative PCR (qPCR) was conducted by using Maxima SYBR Green/Rox qPCR Master Mix (Thermo Scientific, Carlsbad, CA, USA) with the following primers: Twist2, forward: $5^{\prime}-$ TGGACCAAGGCTCTCAGAACA-3' and reverse: 5'-ACAGGAGTATGCGGGCAAGA-3'; Nur77, forward: 5'-CCTGTT GCTAGAGTCTGCCTTC-3' and reverse: 5'-CAATCCAATCACCAAAGCCACG-3'; and Nor-1, forward: 5'-AGACGCCGAAACCGATGT-3' and reverse: 5'-CTCG GACAAGGGCATTCA-3'

ChIP, immunocytochemistry, and EMSA. For ChIP assay, samples were prepared from thymocytes and 16610D9 cells, and immunoprecipitation was performed using anti-Twist2 (3C8, Abnova, Taipei, Taiwan), anti-HDAC7 (H-273x, Santa Cruz Biotechnology, Santa Cruz, CA, USA), anti-p300 (C-20, Santa Cruz Biotechnology) or anti-NFATc3 (F-1, Santa Cruz Biotechnology) antibodies as previously described. ${ }^{52}$ Incubation with anti-IgG antibody or without antibody (NoAb) was used as a negative control for antibody specificity. DNA was purified with a QIAquick Spin Kit (Qiagen, Hilden, Germany) and eluted DNA was analyzed by PCR with the following primers: Nur77 promoter, forward: $5^{\prime}$-GCGGGCAC GGATTTACAACA CC-3' and reverse: 5'-GGGTTCCATTGACGCAGGGAG-3', Nor-1 promoter, forward: $5^{\prime}$-CCCACCCTCCAAGAAGAAGTGAAGC-3' and reverse: 5'-CCCACCCTCCAAGAAGAAGTGAAGC-3', and Twist2 promoter, forward: 5'GCCGAAAAAGCGGAGACAAAACTG-3' and reverse: 5'-GTCGCTCTCAAAGG CTCTGATTTCG-3'. Quantification of PCR band intensity was calculated using the ImageJ software (National Institutes of Health, Bethesda, MD, USA) and normalized to Input. ${ }^{53}$ Immunocytochemistry was performed as described previously. ${ }^{54}$ Carl Zeiss confocal fluorescence microscopy (LSM 710, Zeiss, Oberkochen, Germany) and AxioVision software (Zeiss) were used for detection and analysis. EMSA was performed as described previously. ${ }^{55}$ In brief, $5 \mu \mathrm{g}$ of nuclear extract was incubated with $5 \times$ EMSA-binding buffer and ${ }^{32} \mathrm{P}$-labeled oligonucleotides of c3-1 (sense strand (ss); 5'-CGAAAAAAGCGGAGACAAAACTGAAAGTGC-3'), c3-2 (ss; $5^{\prime}$-GA AGGCCCCAGAATTTGTCCTGCCCCTG-3'), and c3-3 (ss; 5'-GCTCCTGACAGCT ATTTAGCAACCCAGCCG-3').

Bimolecular fluorescence complementation (BiFC) analysis. The principle of BiFC is described elsewhere. ${ }^{36}$ Briefly, HDAC7, MEF2D, Twist2, and Twist2C $\Delta$ were inserted into either VN-173 or VC-155 vector. 293T cells were transfected with a combination of VN/VC vectors. Fluorescence was detected by using the fluorescence microscope (LSM 710).

Statistical analysis. Statistical analysis was performed using Prism 5.0b (GraphPad software, San Diego, CA, USA). Two-tailed Student's $t$-test were used to compare the statistical significance of differences between the samples and their respective controls. The $P$-values are represented in the figures by asterisks $\left({ }^{*} P<0.05,{ }^{* *} P<0.01\right.$, and $\left.{ }^{* *} P<0.001\right)$. The absence of an asterisk or the mark of NS (non-significant) indicates that the change relative to control is not statistically significant

\section{Conflict of Interest}

The authors declare no conflict of interest.

Acknowledgements. We thank Gerald R. Crabtree (Stanford University) for providing cDNA of thymocytes from $\mathrm{Cnb1}^{-1-}$ and NFATC1, $\mathrm{C3}^{-1-}$ mice. This work was supported by a grant from the Korea Health Technology R\&D Project through the Korea Health Industry Development Institute (KHIDI), funded by the Ministry of Health and Welfare, Republic of Korea (H114C0311), and by Korea Mouse Phenotyping Project (2014M3A9D5A01073789) and Basic Science Research program (2016R1A2B3013865) through the National Research Foundation of Korea (NRF) funded by the Ministry of Science, ICT and Future Planning (MSIP). SO, JO, SO, JC, SJ, SH, and YL are supported by the BK21 Plus Program (F15SN01D1305) from the Ministry of Education and NRF. 


\section{Author contributions}

$\mathrm{SO}, \mathrm{JO}, \mathrm{CL}$, and RHS designed research; SO, JO, CL, SO, JC, YL, and SH performed research; SO, JO, CL, and RHS analyzed data; SJ, HL, and RHS generated Twist2 mice; and $\mathrm{SO}, \mathrm{JO}, \mathrm{CL}$, and RHS wrote the paper.

1. Dower NA, Stang SL, Bottorff DA, Ebinu JO, Dickie P, Ostergaard HL et al. RasGRP is essential for mouse thymocyte differentiation and TCR signaling. Nat Immunol 2000; 1 : 317-321.

2. Feske S. Calcium signalling in lymphocyte activation and disease. Nat Rev Immunol 2007; 7 : 690-702.

3. Rincon M, Flavell RA, Davis RA. The JNK and P38 MAP kinase signaling pathways in T cellmediated immune responses. Free Radic Biol Med 2000; 28: 1328-1337.

4. Srikanth $S$, Gwack Y. Orai1-NFAT signalling pathway triggered by T cell receptor stimulation. Mol Cells 2013; 35: 182-194.

5. Cante-Barrett K, Winslow MM, Crabtree GR. Selective role of NFATc3 in positive selection of thymocytes. J Immunol 2007; 179: 103-110.

6. Gong Q, Cheng AM, Akk AM, Alberola-lla J, Gong G, Pawson T et al. Disruption of T cell signaling networks and development by Grb2 haploid insufficiency. Nat Immunol 2001; 2: 29-36.

7. Bueno OF, Brandt EB, Rothenberg ME, Molkentin JD. Defective T cell development and function in calcineurin A beta -deficient mice. Proc Natl Acad Sci U S A 2002; 99 9398-9403.

8. Neilson JR, Winslow MM, Hur EM, Crabtree GR. Calcineurin B1 is essential for positive but not negative selection during thymocyte development. Immunity 2004; 20: 255-266.

9. Oukka M, Ho IC, de la Brousse FC, Hoey T, Grusby MJ, Glimcher LH. The transcription factor NFAT4 is involved in the generation and survival of T cells. Immunity 1998; 9: 295-304.

10. Fischer AM, Katayama CD, Pages G, Pouyssegur J, Hedrick SM. The role of erk1 and erk2 in multiple stages of T cell development. Immunity 2005; 23: 431-443.

11. Rincon M, Whitmarsh A, Yang DD, Weiss L, Derijard B, Jayaraj $P$ et al. The JNK pathway regulates the In vivo deletion of immature CD4(+)CD8(+) thymocytes. J Exp Med 1998; 188: 1817-1830.

12. Sabapathy K, Kallunki T, David JP, Graef I, Karin M, Wagner EF. c-Jun NH2-terminal kinase (JNK)1 and JNK2 have similar and stage-dependent roles in regulating T cell apoptosis and proliferation. J Exp Med 2001; 193: 317-328.

13. Calnan BJ, Szychowski S, Chan FK, Cado D, Winoto A. A role for the orphan steroid receptor Nur77 in apoptosis accompanying antigen-induced negative selection. Immunity 1995; 3: 273-282.

14. Woronicz JD, Calnan B, Ngo V, Winoto A. Requirement for the orphan steroid recepto Nur77 in apoptosis of T-cell hybridomas. Nature 1994; 367: 277-281.

15. Woronicz JD, Lina A, Calnan BJ, Szychowski S, Cheng L, Winoto A. Regulation of the Nur77 orphan steroid receptor in activation-induced apoptosis. Mol Cell Biol 1995; 15: 6364-6376.

16. Weih F, Ryseck RP, Chen L, Bravo R. Apoptosis of nur77/N10-transgenic thymocytes involves the Fas/Fas ligand pathway. Proc Natl Acad Sci U S A 1996; 93: 5533-5538.

17. Cheng LE, Chan FK, Cado D, Winoto A. Functional redundancy of the Nur77 and Nor-1 orphan steroid receptors in T-cell apoptosis. EMBO J 1997; 16: 1865-1875.

18. Lee SL, Wesselschmidt RL, Linette GP, Kanagawa O, Russell JH, Milbrandt J. Unimpaired thymic and peripheral T cell death in mice lacking the nuclear receptor NGFI-B (Nur77). Science 1995; 269: 532-535.

19. Zhou T, Cheng J, Yang P, Wang Z, Liu C, Su X et al. Inhibition of Nur77/Nurr1 leads to inefficient clonal deletion of self-reactive T cells. J Exp Med 1996; 183: 1879-1892.

20. Dequiedt F, Kasler H, Fischle W, Kiermer V, Weinstein M, Herndier BG et al. HDAC7, a thymus-specific class II histone deacetylase, regulates Nur77 transcription and TCR-mediated apoptosis. Immunity 2003; 18: 687-698.

21. Dequiedt F, Van Lint J, Lecomte E, Van Duppen V, Seufferlein T, Vandenheede JR et al. Phosphorylation of histone deacetylase 7 by protein kinase $D$ mediates $T$ cell receptorinduced Nur77 expression and apoptosis. J Exp Med 2005; 201: 793-804.

22. Parra M, Kasler H, McKinsey TA, Olson EN, Verdin E. Protein kinase D1 phosphorylates HDAC7 and induces its nuclear export after T-cell receptor activation. J Biol Chem 2005; 280 : 13762-13770.

23. Youn HD, Chatila TA, Liu JO. Integration of calcineurin and MEF2 signals by the coactivator p300 during T-cell apoptosis. EMBO J 2000; 19: 4323-4331.

24. Blaeser F, Ho N, Prywes R, Chatila TA. Ca(2+)-dependent gene expression mediated by MEF2 transcription factors. J Biol Chem 2000; 275: 197-209.

25. Kasler HG, Victoria J, Duramad O, Winoto A. ERK5 is a novel type of mitogen-activated protein kinase containing a transcriptional activation domain. Mol Cell Biol 2000; 20: 8382-8389.

26. Kasler HG, Young BD, Mottet D, Lim HW, Collins AM, Olson EN et al. Histone deacetylase 7 regulates cell survival and TCR signaling in CD4/CD8 double-positive thymocytes. J Immunol 2011; 186: 4782-4793.

27. Bain G, Quong MW, Soloff RS, Hedrick SM, Murre C. Thymocyte maturation is regulated by the activity of the helix-loop-helix protein, E47. J Exp Med 1999; 190: 1605-1616.

28. Masuda ES, Naito Y, Tokumitsu H, Campbell D, Saito F, Hannum C et al. NFATx, a novel member of the nuclear factor of activated $T$ cells family that is expressed predominantly in the thymus. Mol Cell Biol 1995; 15: 2697-2706.
29. Wilkinson B, Kaye J. Requirement for sustained MAPK signaling in both CD4 and CD8 lineage commitment: a threshold model. Cell Immunol 2001; 211: 86-95.

30. Shao H, Wilkinson B, Lee B, Han PC, Kaye J. Slow accumulation of active mitogen-activated protein kinase during thymocyte differentiation regulates the temporal pattern of transcription factor gene expression. J Immunol 1999; 163: 603-610.

31. Davey GM, Schober SL, Endrizzi BT, Dutcher AK, Jameson SC, Hogquist KA. Preselection thymocytes are more sensitive to T cell receptor stimulation than mature T cells. J Exp Med 1998; 188: 1867-1874.

32. Staton TL, Lazarevic V, Jones DC, Lanser AJ, Takagi T, Ishii S et al. Dampening of death pathways by schnurri-2 is essential for T-cell development. Nature 2011; 472: 105-109.

33. Fassett MS, Jiang W, D'Alise AM, Mathis D, Benoist C. Nuclear receptor Nr4a1 modulates both regulatory T-cell (Treg) differentiation and clonal deletion. Proc Natl Acad Sci U S A 2012; 109: 3891-3896.

34. Kasler HG, Lim HW, Mottet D, Collins AM, Lee IS, Verdin E. Nuclear export of histone deacetylase 7 during thymic selection is required for immune self-tolerance. EMBO J 2012; 31: 4453-4465.

35. Chen J, Fiskus W, Eaton K, Fernandez P, Wang Y, Rao R et al. Cotreatment with BCL-2 antagonist sensitizes cutaneous T-cell lymphoma to lethal action of HDAC7-Nur77-based mechanism. Blood 2009; 113: 4038-4048.

36. Song YB, Park CO, Jeong JY, Huh WK. Monitoring G protein-coupled receptor activation using an adenovirus-based beta-arrestin bimolecular fluorescence complementation assay. Anal Biochem 2014; 449: 32-41.

37. Gong XQ, Li L. Dermo-1, a multifunctional basic helix-loop-helix protein, represses MyoD transactivation via the HLH domain, MEF2 interaction, and chromatin deacetylation. J Biol Chem 2002; 277: 12310-12317.

38. Chow CW, Rincon M, Cavanagh J, Dickens M, Davis RJ. Nuclear accumulation of NFAT4 opposed by the JNK signal transduction pathway. Science 1997; 278: 1638-1641.

39. Sosic D, Richardson JA, Yu K, Ornitz DM, Olson EN. Twist regulates cytokine gene expression through a negative feedback loop that represses NF-kappaB activity. Cell 2003; 112: 169-180.

40. de Kossodo S, Cruz PD Jr., Dougherty I, Thompson P, Silva-Valdez M, Beutler B. Expression of the tumor necrosis factor gene by dermal fibroblasts in response to ultraviolet irradiation or lipopolysaccharide. J Invest Dermatol 1995; 104: 318-322.

41. Page DM, Roberts EM, Peschon JJ, Hedrick SM. TNF receptor-deficient mice reveal striking differences between several models of thymocyte negative selection. J Immunol 1998; 160: 120-133.

42. Baseta JG, Stutman O. TNF regulates thymocyte production by apoptosis and proliferation of the triple negative (CD3-CD4-CD8-) subset. J Immunol 2000; 165: 5621-5630.

43. Li L, Cserjesi P, Olson EN. Dermo-1: a novel twist-related bHLH protein expressed in the developing dermis. Dev Biol 1995; 172: 280-292.

44. Rivera RR, Johns CP, Quan J, Johnson RS, Murre C. Thymocyte selection is regulated by the helix-loop-helix inhibitor protein, Id3. Immunity 2000; 12: 17-26.

45. Lee YS, Lee HH, Park J, Yoo EJ, Glackin CA, Choi YI et al. Twist2, a novel ADD1/SREBP1c interacting protein, represses the transcriptional activity of ADD1/SREBP1c. Nucleic Acids Res 2003; 31: 7165-7174.

46. Moran AE, Holzapfel KL, Xing Y, Cunningham NR, Maltzman JS, Punt J et al. T cell receptor signal strength in Treg and iNKT cell development demonstrated by a novel fluorescent reporter mouse. J Exp Med 2011; 208: 1279-1289.

47. Baldwin TA, Hogquist KA. Transcriptional analysis of clonal deletion in vivo. J Immunol 2007; 179: 837-844.

48. Hu QN, Baldwin TA. Differential roles for Bim and Nur77 in thymocyte clonal deletion induced by ubiquitous self-antigen. $J$ Immunol 2015; 194: 2643-2653.

49. Chaffin KE, Beals CR, Wilkie TM, Forbush KA, Simon MI, Perlmutter RM. Dissection of thymocyte signaling pathways by in vivo expression of pertussis toxin ADP-ribosyltransferase. EMBO J 1990; 9: 3821-3829.

50. Han S, Choi H, Ko MG, Choi YI, Sohn DH, Kim JK et al. Peripheral T cells become sensitive to glucocorticoid- and stress-induced apoptosis in transgenic mice overexpressing SRG3. J Immunol 2001; 167: 805-810.

51. Kim Y, Kim H, Jeoung D. Tubulin Beta3 Serves as a Target of HDAC3 and Mediates Resistance to Microtubule-Targeting Drugs. Mol Cells 2015; 38: 705-714.

52. Choi J, Jeon S, Choi S, Park K, Seong RH. The SWI/SNF chromatin remodeling complex regulates germinal center formation by repressing Blimp-1 expression. Proc Natl Acad Sci U S A 2015; 112: E718-E727.

53. Kwon YH, Bishayee K, Rahman A, Hong JS, Lim SS, Huh SO. Morus alba Accumulates Reactive Oxygen Species to Initiate Apoptosis via FOXO-Caspase 3-Dependent Pathway in Neuroblastoma Cells. Mol Cells 2015; 38: 630-637.

54. Feske S, Draeger R, Peter HH, Eichmann K, Rao A. The duration of nuclear residence of NFAT determines the pattern of cytokine expression in human SCID T cells. J Immunol 2000; 165: 297-305.

55. Ko M, Ahn J, Lee $\mathrm{C}$, Chung $\mathrm{H}$, Jeon $\mathrm{SH}$, Chung HY et al. E2A/HEB and Id 3 proteins control the sensitivity to glucocorticoid-induced apoptosis in thymocytes by regulating the SRG3 expression. J Biol Chem 2004; 279: 21916-21923. 